C-A/AP/\#288

August 2007

\title{
AGS BPM Tests
}

P. Menga, L. Ahrens, R. Schroeder

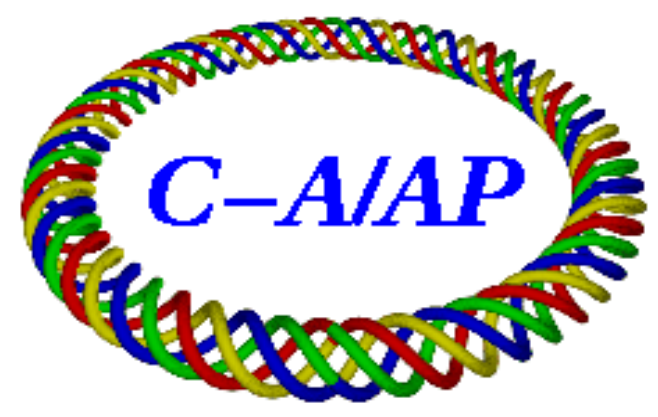

Collider-Accelerator Department Brookhaven National Laboratory Upton, NY 11973 
Introduction:

\section{AGS BPM Tests}

The AGS BPM's were tested over the summer shutdown of 2006. The testing procedure, results, equipment changes, and modifications to the test will be explained here. As a result of this work we restored 12 out of the 140 planes that make up the system - a significant gain. There remain ten planes which do not work for reasons unknown and 8 planes that new need electronics to function. A detailed description of the effectiveness of the tests is found at the end of the main text. We will begin with a description of the setup and construction of the AGS BPM system.

\section{AGS BPM background - Signal Collecting and Hardware:}

A pickup electrode (PUE) is a device inside the beam pipe. In the case of the AGS, this is of the electrostatic (also called capacitive) variety. This is as opposed to the button PUE, normally recessed into the beam pipe and optimized to respond to the high frequency components of the bunch transverse position (normally used for electron and positron beams), and the directional coupled PUE, where a resonance wave is formed on the electrode.

We shall examine the characteristics of the PUE. The current induced on the inner wall of the beam pipe by a centered beam is azimuthally constant. If the beam is off-center, the electric field - and hence the induced charge-will be strongest nearest to the beam. So suppose we placed two parallel plates inside the beam pipe, in the same plane and perpendicular to that plane. These plates will intercept the electric field from the beam, and the amount of field on each plate will let us know the beam position. For a centered beam, both plates would intercept an electric field of the same strength.

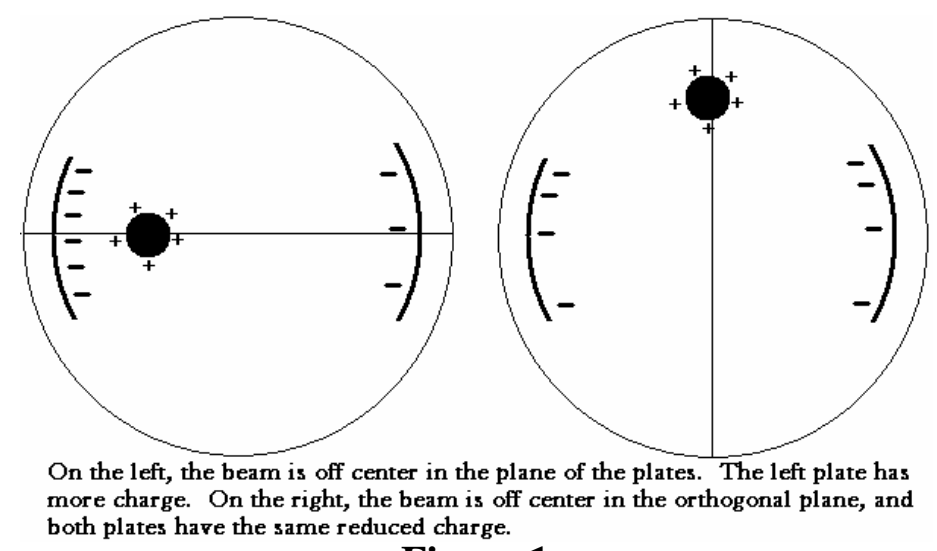

Figure 1

If the beam was off center, in the same plane as the plates, the closer plate would have a larger induced charge than the other. Our position measurement is extracted by subtracting the induced charge (or voltage) of one plate from the other. If the beam was 
off center in the opposite plane, both plates should experience the same field, though the magnitude would decrease. From this we see that the difference signal should be divided by the sum signal for normalizing purposes. We also would not be able to tell in which direction the beam had moved if it was off center in the opposite plane. Therefore, in order to completely describe position in the transverse plane, we see the need for two pairs of plates in an orthogonal layout.

Refer to drawing D06-M-RD1783-5, Appendix II, for the exact layout of the AGS PUE's. The plates are angled, instead of being rectangular, to improve the linearity of the measured position. The output remains reasonably linear for offsets small compared to the beam pipe radius. The normalizing factor in the above discussion now needs to be the sum of all four electrodes and there is a new scaling factor needed (see AGS Tech Note \# 459 for experimental data). The electrodes will only detect bunched beam, not a DC beam. Though it does not discuss the four plate geometry exactly, the following paper provides a good mathematical background: "Beam Position Monitoring", Robert Shafer, AIP Conference Proceedings 212: Accelerator Instrumentation, NY 1989.

Two electrode plates are on either side of the beam pipe vertically (numbered 1 and 2 ) and two are on either side horizontally ( 3 and 4 ). They occur at the following downstream positions in every superperiod: $2,4,8,12,14$, and 18, which are points of average dispersion and beta in the optically undistorted machine. All four cables from the electrodes exit the vacuum chamber pointing either to the inside or outside of the ring, dependent upon position. The AGS main dipole magnets are shaped like 'C's when viewed down the longitudinal axis. For the first half of a superperiod (magnets $1-10$ ), the ' $C$ ' opens inward; for the second half $(11-20)$, the ' $C$ ' opens outward. The beam pipe ion vacuum pumps are always placed on the side of the magnet with the backleg, or vertical part of the ' $\mathrm{C}$ '. Hence there is a physical space concern, so the PUE must be placed on the other side - the opening of the ' $\mathrm{C}$ '.

From the PUE, an EL (curved piece of pipe) carries the signal cables outward. This pipe curves in the horizontal plane, with respect to the ring. This EL is on the upstream side of the PUE (see drawing D06-M-818-5, Appendix III). The same assembly is used for PUE's that point in or out, with a rotation of $180^{\circ}$. Therefore the vertical plates stay vertical, but 1 and 2 will be swapped; 3 and 4 will be swapped for the horizontal. This assembly is not perfectly symmetric, due to the output wiring. So there is a structure: 2,4, and 8 are the same but opposite from 12,14, and 18 as explained above.

Sitting on a platform near the beam pipe by every electrode is a matchbox. A single matchbox receives all four cables. The purpose of the matchbox is to flatten the frequency response of the electrodes in the band of interest, about $1 \mathrm{MHz}$ to $10 \mathrm{MHz}$. Ideally, there would be no coupling among the four channels in the matchbox. The output then travels along solid shield coax cable outside the ring to one of three houses. Superperiods K, L, A, and B run to the A10 house; C through F to the E10 house; and G through $\mathrm{J}$ to the H10 house. The electrical length of each set of four cables is matched in order to eliminate any phase shifts between them. Grounding is not done in the houses, something one must be careful to maintain, but through the ring using a single point grounding system. Inside each house, the cables from each electrode connect to an 
electronic chassis isolated from all the other BPM's. Here, the sums and differences of the signals are taken, in a manner to be described later. Each electronic chassis also receives the local oscillator signal (LO) from the low-level AGS RF system in order to demodulate the PUE signal from the beam spectrum.

The last step in the electronics is a voltage to frequency converter. The frequency encoded voltage signal is sent from the chassis to a patch panel via a fiber-optic cable. The patch panel collects the fiber optics from a whole superperiod and connects them with further equipment in Bldg 929. Some of this equipment will be discussed later. The final step is the display of the data in a linux program called AgsOrbitDisplay, available from the StartUp application.

\section{Software:}

AgsOrbitDisplay has several settings of interest. First it should be noted that it is imperative to set it to the correct PPM user, otherwise it will not take any data. An 'electrode file' is used to set which BPM's are 'bad' and hence ignored, as well as what the offset correction and gain factor should be for each BPM. For all our testing we used the 'CalibrateOld' electrode file, which did not flag any BPM's as bad and had an offset of zero and gain of one for every BPM. For every cycle of the AGS main magnet, data can be taken at multiple times. This is configured through the 'ScanTimes...' button. For all our tests we looked at times of 50, 100, 150, .., 600, 650, and $700 \mathrm{~ms}$ from T0 (a total of 14 times). The beam frequency changed over these times, allowing us to inspect the frequency dependence of the BPM's if we wished. Collecting data at multiple times as opposed to a single time did not cost us any 'real' time during the measurements.

What would cost real time is how many cycles are collected, set in the 'No.Cycles:' field. At first we looked at five cycles, but later increased this to 15. An AGS cycle being approximately four seconds, this caused data acquisition to run about a minute. When looking at multiple cycles and times, the program would prefer to display each cycle and each time. Since this can take a very long time to draw graphically, it is expedient to do the following: set the 'Avg:' field to 'Yes' and select only one time to examine in the 'DispTimes:' field.

The program rejects entire cycles were one measured intensity is less than a threshold, and since many of the reported intensities are quite low, it is necessary to set the 'Threshold:' field to zero to bypass this feature. The 'Graph:' type we used was 'Data<> Loc' (data vs. ring location).

Averaging the cycles also had another great benefit for our tests. One of us, LA, was concerned about the variations in reported position from one cycle to the next. When we let the program average the cycles and had 'Sigmas:' set to 'Yes', the displayed graph showed a vertical bar indicative of each BPM's sigma, or cycle-to-cycle variation. Using the 'Data' pull down menu and the selection 'List Data', a table of numerical offsets and sigmas would be generated. The form of this table was dependent on the selections for 'Ave:' and 'Sigmas:', as well as which graphs were displayed. Normally we chose to display all three, the 'DataType:' field set to 'HorVer\&Int'. 
For every test we did, the data was saved. MPM used a certain syntax to name the files, which will be explained later. The data was voluminous, so I wrote a series of $\mathrm{C}++$ programs to extract the relevant data. Apparently AgsOrbitDisplay saved the data in a variety of ways. If from within the program one opened up a saved file, the data (unaveraged) for every cycle and time was available. Yet it was discovered that a file was also saved to /home/cfsb/mcr/AgsBpms/ in the linux system, under the same name, that just recorded the average offset and sigma for every BPM at every time. This was the file I used. The syntax of this file is explained below.

There are three blocks of data, the first for horizontal, the next vertical, and the last intensity. All blocks use the same syntax so just the first will be explained. The first time data was taken, which may differ from the requested time by microseconds, is listed. Following this is 72 rows with two columns. The first column is average value (either offset or intensity) and the second is the calculated sigma. The 72 rows correspond to the 72 possible BPM's-A2, A4, .., L14, and L18. Then the next time data was taken is listed, followed by another 72 rows. After all the horizontal times and data have been listed, the file begins with the first time and data for the vertical, with no obvious marker except the reset of time. Since we used a fixed number of times (14), this was easy to account for. The intensity data was then displayed in the same manner. A program to pick out the proper data from such a file is simple to engineer.

The Electronics:

Before we discuss the testing, a brief word is due on the electronics (Appendix IV). If you look at the PUE signal broadband (all significant harmonics are included) on a scope (directly at the plates and with a high input impedance), you see something that has the shape of the bunch in time. If you filter the PUE signal and select only a particular harmonic of interest, you see a sine wave with a time dependent amplitude and phase. The amplitude of that sine wave depends on the beam position relative to a PUE plate, the beam intensity (current) and the shape of the bunch. The BPM processing electronics are only interested in narrowband $(\sim 20 \mathrm{kHz} \mathrm{BW})$ processing of a specific spectral component - the $2^{\text {nd }}$ harmonic of the accelerating RF. [Kevin Smith, AGS \& Booster System Random Bits of Information, (10/9/03). See Appendix V]

The RF frequency, when the system is setup to accelerate on the 12th harmonic of the revolution frequency of the beam, is normally around 4 to $4.5 \mathrm{Mhz}$ (before bunch merging came about). Therefore the second harmonic is about 8 to $9 \mathrm{Mhz}$. The LO signal sent to the electronics is about $29.9 \mathrm{MHz}\left(21.4 \mathrm{MHz}\right.$ plus $2^{\text {nd }}$ harmonic). The first step in the electronics is to "mix" the PUE signal with the LO to extract the $2^{\text {nd }}$ harmonic and up-convert to a fixed frequency, 21.4 MHz. The next stage looks at electrode pairs 1 $\& 2$ and $3 \& 4$. Sums and differences of these pairs are taken. The two sums are then summed. After amplification and more mixing, we have the following:

$$
\begin{aligned}
& \text { Horizontal Out } \propto \frac{(3-4)}{1+2+3+4} \\
& \text { Vertical Out } \propto \frac{(1-2)}{1+2+3+4} \\
& \text { Intensity Out } \propto 1+2+3+4
\end{aligned}
$$


There is no guarantee that the numerators may not actually be (4-3) or (2-1) due to the rotation of the PUE around the longitudinal axis (see page 2. The connections to the electronics are labeled IN, OUT, UP, and DOWN — not by PUE plate number). The final step in the electronics is to convert the voltage corresponding to the above into a frequency, and output that frequency via fiber optic cable. It is important to note that the intensity channel could be working fine for determining $\mathrm{H}$ and $\mathrm{V}$ position but, due to a failure in the voltage to frequency converter or fiber optic transmitter, may not be read correctly by the software. That is, an apparently broken intensity signal does not necessarily mean a broken BPM. (Some fiber optic transmitters have been taken from intensity channels to fix $\mathrm{H}$ and $\mathrm{V}$ channels).

Again from Kevin Smith [10/9/03], we have the following technicalities: AGS analog signals (in the BPM chassis) are $+/-1 \mathrm{~V}=+/$ - full aperture. Fiber optic signals are encoded such that analog $0 \mathrm{~V}=9.0 \mathrm{MHz}=$ center aperture, and analog $+/-1 \mathrm{~V}$ $=+/-1 \mathrm{MHz}(10$ or $8 \mathrm{MHz})=+/$ - full aperture. For intensity, $-.6 \mathrm{~V}=\min ,+.6 \mathrm{~V}=\max$, $? \mathrm{~V}=.2 \mathrm{~V}=$ factor of ten in intensity (logarithmic) and fiber optic signals are encoded such that analog $0 \mathrm{~V}=$ mid intensity $=9 \mathrm{MHz}$, and analog $+/-.6 \mathrm{~V}=+/-.6 \mathrm{MHz}$ (9.6 or $8.4 \mathrm{MHz})=\max / \min$ intensity .

\section{The Test Method:}

The physicist had noticed during previous AGS runs that various of the BPM's seemed to give bad data. The most prominent sign of this was a railed signal. In addition, some electrodes appeared to respond to the beam but the measurement would bounce around from cycle to cycle, while the BPM's on either side were reporting a constant measurement.

The following BPM's were reported as bad in electrode file 'OffsetsFrom2003Survey':

\begin{tabular}{|l|l|}
\hline $\mathrm{A} 02 \mathrm{H}$ & F02 V \\
\hline $\mathrm{A} 08 \mathrm{H}$ & F12 H \\
\hline $\mathrm{A} 18 \mathrm{~V}$ & F12 V \\
\hline B04 H & F14 V \\
\hline B04 V & J02 H \\
\hline B12 V & J02 V \\
\hline E04 H & J14 V \\
\hline E04 V & K18 H \\
\hline
\end{tabular}

The following did not have electronic chassis in the houses:

\begin{tabular}{|l|l|}
\hline B08 & C12 \\
\hline B18 & F08 \\
\hline C04 & G14 \\
\hline
\end{tabular}

Historically, BPM channels marked as bad during machine operations have been subsequently tested by one method or another and found to be functioning properly. Then when beam became available the channels would again malfunction. This previous testing concentrated mainly on components. We judged it prudent to try a broad test on the whole system. 
It was decided an independent test method should be used to verify that these BPM's were indeed bad, try to find any other suspect ones, and help in developing a plan to fix them. For the BPM's in the Booster, there is a calibration ring around each electrode set. A voltage can be applied to this ring to induce a charge on the electrodes that can then be measured for testing. This calibration ring is absent for the AGS electrodes. Another good method is to use a cable centered in the beam pipe with a current on it to simulate beam conditions. Unfortunately, we could not open up the beam pipe to do such a measurement during the summer shut down. In fact, this test will probably never be done here due to time and budget constraints. So, in the absence of real beam, the following method was evolved.

To test, a sine wave of beam frequency was used to "tickle" two of the plates (in the same plane). This signal was sent from within a house to go through only the matchbox and electrode. This signal induced some charge on the plates and created an electric field inside the beam pipe. This field in turn induced a charge on the plates of the other plane, which was then measured using the entire apparatus as explained above.

\section{Bench Measurements:}

Before commencing the testing of the ring BPMs, a "bench measurement" was carried out to check that there was a good chance the method would generate sufficient signal to be useful. This measurement was made using one of the spare AGS vacuum chamber assemblies stored at building 919B. A few of these vacuum chambers include pickup electrodes (PUE) assemblies which, given that the PUE is located at one end of the vacuum chamber, are easily accessed. Each of the four plates that make up a PUE assembly and the common ground are available via standard BNC connectors on the chamber. The plates themselves, and the spaces between them, are also easily accessed, which allows some simple checks to be carried out. For example, if we are measuring an effect due to the capacitance between these plates then the effect should change if the material in the space is changed.

The measurement involved applying a pulse with fast ( 10 ns) rise and fall time, and with several 100's of ns width, to one of the plates and observing any response from the other plates. The output-viewing scope channel was set to high (1 MO) impedance. The applied pulse also went past a high impedance scope input on its way into the PUE plate. The input pulse had an amplitude of $24.2 \mathrm{~V}$. The two adjacent plates each reported $(90+/-5) \mathrm{mV}$. The plate across from the driven plate reported $(27+/-3) \mathrm{mV}$. These induced pulses were observed to decay with a time constant of roughly $190 \mu \mathrm{s}$. This pattern of induced pulses was rather insensitive to which plate was chosen as the driving plate.

From this some conclusions can be drawn. The decay time together with the 1 MO resistance imply a capacitance of $\left(190 ? 10^{-6}\right) /\left(10^{6}\right)=190 \mathrm{pF}$. This is reasonable for the plate and internal connecting wire (expect $\sim 100 \mathrm{pF})+$ the scope $(20 \mathrm{pF})+$ the cable. What we want is the implied capacitance coupling the driven plate to the others. The ratio of this coupling capacitance to the just measured total plate capacitance is equal to the ratio of the input voltage to the output voltage. From this we expect the coupling to 
adjacent plates to be about $0.7 \mathrm{pF}$ and the coupling across the assembly to the far plate to be about $0.2 \mathrm{pF}$.

The electronics of the AGS equilibrium orbit system are quite sensitive. We would expect to be able to see a bunch with an intensity of $10^{9}$ protons. So what voltage in our pulser would we need to mock up a bunch of $10^{9}$ protons? About $1 \%$ of the bunch is inside the plates at any time during its passage. We have a 5 inch plate, and since the bunch width is about $50 \mathrm{~ns}$ and its velocity is roughly 12 inches/ns, then $50 \mathrm{~ns}$ ? 12 inches $/ \mathrm{ns}=600$ inches. So plate length $/$ bunch length $=5 / 600 \sim 1 \%$. This charge gets split among the four plates almost equally. So, with $1 \%$ of $1 / 4$ of the total protons, we need to induce a charge of $2.5 ? 10^{6}$ protons or $4 ? 10^{-13}$ Coulombs on our plate. The charge induced is the voltage applied $*$ the coupling capacitance or $\mathrm{V} *(0.7 \mathrm{pF})$. To get 4 ? $10^{-13}$ $\mathrm{C}$ we need $\left(4 ? 10^{-13}\right) /\left(0.7 ? 10^{-12}\right)=0.5 \mathrm{~V}$. If we could apply $0.5 \mathrm{~V}$ to the drive plate in a format resembling a bunch we should introduce a measurable signal into the system. This turned out to be feasible. We call this 'tickling'.

\section{The Measurements:}

There were several variations of the tickle test, and I shall describe the evolution of the methods we used, to guide further refinements. Actual BPM testing began on $10 / 12 / 06$. Here, we were trying to develop our test method and verify that we knew how to use it. Data for this day is saved in the elog: 'Booster-AGS-pp_2007 Thu_Oct_12_2006_9:29:01_AM'. Here we notice several important things. When no signal is sent to a BPM, the data displayed normally shows a large sigma (around 4 or more). By not averaging the cycles but displaying every one, this is a very easy effect to see. When we were testing a particular plane of a certain BPM, the data converged at that BPM. See figure 2, E14 (remember the BPM's are at positions 2, 4, 8, 12, 14, and 18).

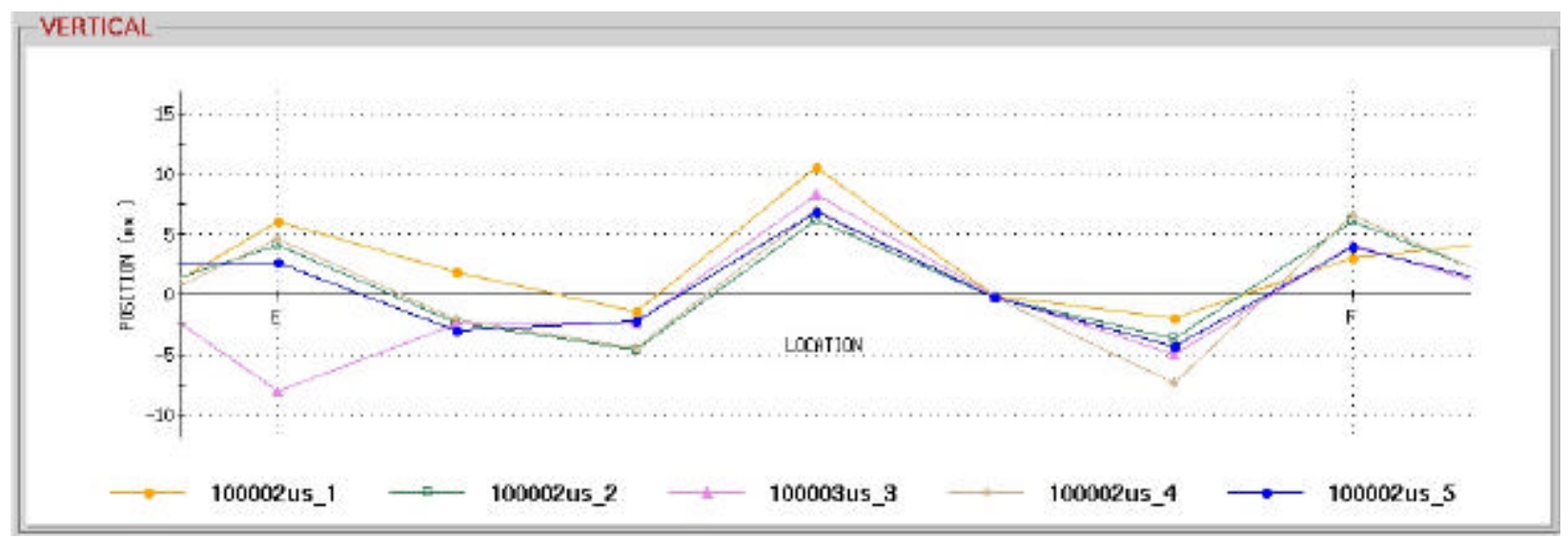

Figure 2

Here was the test we tried: input our signal into plate \#1 and disconnect plate \#2 from the electronics, and then connect plates \#3 and \#4 into any one of three states: both connected, and then one or the other disconnected. This would then be repeated with the roles of $1 \& 2$ and $3 \& 4$ swapped. If plate 1 was symmetrically coupled to the adjacent plates, with both 3 and 4 connected we would expect to see a zero horizontal offset with no variation. Then with either 3 or 4 disconnected, we should see a positive or negative 
offset of similar absolute value. We should expect very similar results if we went in on 2 instead of 1 .

We did not observe this, so we judged that we did not have perfectly symmetric coupling. For example, at E12 we got fairly symmetric data $(+/-60 \mathrm{~mm})$ for the disconnected states, but it was not around the zero position when both plates were connected. We worried that we were going into some railed state with one side offperhaps $\sim 60 \mathrm{~mm}$ was the largest value that could be reported. It was decided the test should be modified to include putting in $-3 \mathrm{~dB}$ attenuators between the matchbox and electronics. 'Tickle' test as of 10/18/06:

1: input signal to plate 1, Plate 2 disconnected, 3 and 4 normal (checks horizontal)

2:

3:

4:

5:

6:
, 3 with $-3 \mathrm{~dB}$ attenuation, 4 normal

, 3 normal, 4 with $-3 \mathrm{~dB}$ attenuation

input signal to plate 3 , plate 4 disconnected, 1 and 2 normal (checks vertical)

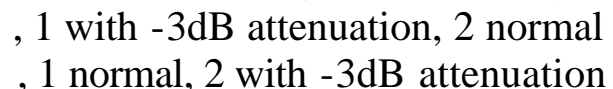

We also looked at the time dependence of the data (frequency sweeps with time and offset is affected by frequency). Frequency sweeps between about $4 \mathrm{MHz}$ and 4.5 $\mathrm{MHz}$ during the time interval from 200 to $600 \mathrm{~ms}$. See figure 3.

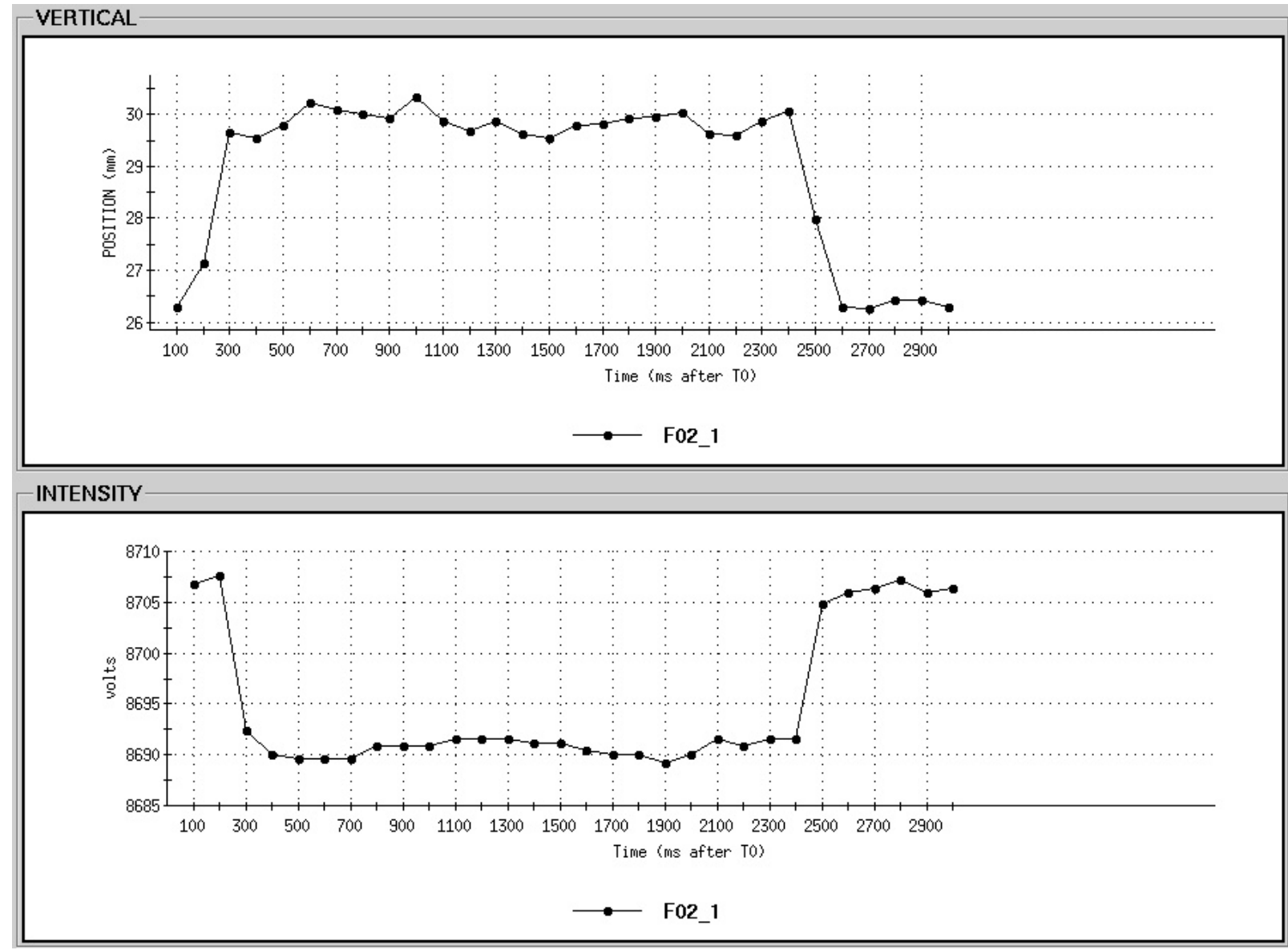

Figure 3 
We used the new method to test all the available BPM's in superperiods A, B, and L. The input signal to the PUE had an RMS amplitude of $380 \mathrm{mV}$. The first thing we discovered was that making six different sets of connections to test every BPM was very time consuming. The second thing we noticed was that the states with the attenuators on either leg did not always produce a symmetric difference from the unattenuated state. Again, this could be due to a 'railing' effect or another problem.

The first system we found suspect was B12. The B12 Vertical channel reported an offset of about $72 \mathrm{~mm}$ no matter how we connected the attenuators. This prompted us to perform what will henceforth be called an electronics test. For this we sent our signal directly into the channel in question on the electronic chassis. For the vertical, say, a splitter was used to put the signal into both channels $1 \& 2$. Then an attenuator would be put alternately into either channel. For this we used selectable attenuators (0 to $11 \mathrm{db})$ for both channels. For B12 V, this showed the electronics to be okay.

The filename syntax used for saved AgsOrbitDisplay files was the following: "BPM name, signal in channel, $3 \mathrm{~dB}$ connector channel (if any)". For example, the test on B2 where the signal went in plate 1 and we had an attenuator on channel 3 was saved as "B02,1,3". For electronics tests, "TEST" was inserted after the name of the BPM, e.g. "B12TEST,1-2,1." Notice since the signal was fed to $1 \& 2$, both numbers and a hyphen are used.

After this data was taken, it was converted to an Excel spreadsheet and several graphs were made. Though the graphs are not included, the important inferences will be given. There are general trends that:

* Offset decreases with intensity

* Sigma decreases with intensity

* Hence, sigma decreases with smaller offsets

* Later it was seen that if the offset really is on a 'rail', sigma will be small or zero

* The shifts with attenuators seemed to be smaller when the initial offset was larger

Due to the time spent performing the test, and the judgment that we needed a small reported offset for the sigma data to be valid, we modified the procedure (10/24/06) and retested everything we had already done and preceded to the rest. The new procedure can be expressed as this (Appendix VI):

1: input signal into plates $1 \& 2$ (checks horizontal)

2: input signal into plates $3 \& 4$ (checks vertical)

3: if either of the above did not lead to an offset of less than $45 \mathrm{~mm}$ (away from the rail) THEN just send the signal into one plate or the other.

4: if any of the data received is bad (low offset not attainable, bad sigma) THEN perform an electronics test.

An analysis of this data will follow, but first I will describe some different tests we found ourselves doing in the AGS ring itself. What might motivate this is that we received bad data from the initial test but the electronics test was okay. Then we might 
ponder the problem lies in the ring. Later it came to our attention the problem could also be with equipment downstream of the electronics, but I will cover that later.

\section{$\underline{\text { Ring Tests: }}$}

The primary tool we used for our testing in the ring (11/3 and 11/6) was a network analyzer (HP E5100A, $300 \mathrm{MHz}$ ). This equipment sends a function of known amplitude out and compares it to the returned signal, the important value calculated being the attenuation. It also displays the phase of the returned signal. We set the frequency of the sent function to be $8.5 \mathrm{MHz}$ and used it to test the matchbox and electrode together, and each by itself. For both devices together or just the electrode we recorded four data points: 1 to 3,1 to 4,2 to 3 , and 2 to 4 . For just the matchbox, we went in one side and out the other on the same channel.

From this test we determined that the electrodes by themselves were all very similar in the four configurations and showed drops within $1 \mathrm{~dB}$ of $-50 \mathrm{~dB}$. The matchboxes by themselves were also very similar on every channel, with a loss of about $-1.5 \mathrm{~dB}$. The big surprise came when they were tested together. A2 for example, showed a total difference of $20 \mathrm{~dB}$ between certain channels. This has led to the suspicion that there is coupling between the four channels inside the matchboxes. Another concern was that the phase data the analyzer gave was always the same for three of the test configurations, but different for the last. The one where it was different was always the same physical hook-up on the matchbox (regardless of the numbering). Kevin Smith says [10/9/03] that the RF signals from each plate are born phase coherent and remain so when they reach the BPM electronics. This test makes us not so sure. On two of the matchboxes, at E4 and F2, this 'odd' phase did not even appear similar to the other 'odd' phases we had observed.

In the process of testing, we found the J14 matchbox to have a loose connection inside which was re-soldered. Tightening the SMA connectors on the box also brought its throughput loss down from $-3 \mathrm{~dB}$ to $-1.5 \mathrm{~dB}$. We also found the $\mathrm{J} 2$ box to have two broken connectors, but was unfixable due to parts needed. We moved some matchboxes around to compensate, as described later. Already concerned about loose connections, we had tightened all wire connections to the matchboxes in the ring before the 'tickle' test of 10/24 was begun. Whether this was worthwhile is hard to judge, though we did find a handful of connections that were very loose.

The Data Analysis:

Hand notes were taken during the tickle tests, but for added precision and computer analysis vagaries, the data was lifted from the saved files using simple $\mathrm{C}++$ data mining programs. The syntax of the saved data is explained near the top of page 4 . After looking at histograms of the data, it was determined by LA that sigmas greater than 1.8 were unusual. MPM made the determination that any absolute offset over 30 was also unusual. Hence, two critical points were determined that separated the wheat from the chaff. Since frequency and thus measurements changed over the 14 times of interest, we applied these critical values to all times. See Appendix I for data. 
The data analyzed in Appendix I was where both plates of a plane were activated. As noted in the procedure, if we received a high offset with this method then we activated just a single plate. Of those listed in the appendix, that helped A02 V, B12 H, F12 H, H4 $\mathrm{V}, \mathrm{J} 2 \mathrm{H}$, and $\mathrm{J} 14 \mathrm{~V}$. Ignoring offsets less than 30, the following were then understood to be bad: A18 V, B12 V, F14 V, and J2 V. The offset of 37 for H2 V was never explored. An important note on the offsets and our method: using the old method (of 10/18), always sending the signal into just one plate, we frequently received bad offsets. That is what led to the new method. The point is, that with the new method, even a BPM that did not show a high offset could still have a high offset if JUST one or the other plates was used. The importance of this is not understood, though the four bad offsets listed above were bad regardless of the connection method.

If we noticed a bad offset or sigma during testing we normally performed an electronics test. Such tests were preformed on the following: A02, B12, F2 H, F12 H, and F14. This showed:

A2: bad offset or sigma NOT due to electronics

B12: bad vertical offset NOT due to electronics, though just the electronics gave a bad offset for the horizontal (not understood). Horizontal sigma due to electronics

F2 H: just a test of the method where we generate the LO, seemed good

F12 H: bad sigma due to electronics (F12 V should have been tested too, but was not)

F14: Vertical offset NOT due to electronics

Other sneaky tests we did on the electronics involved swapping cables. To see if a low reported intensity was due to electronics, we would switch the fiber optic output with nearby electronics. This always showed the problem to be upstream of the fiber optics. This was done for G8, G18, I8, and J14. These files were saved as G08-12TEST, for example, where we switched G08 and G12 fiber optic intensity outputs. For A18, we switched the vertical and horizontal fiber optic outputs and saw the high offset then appeared on the horizontal. We decided the electronics were bad, but found the real cause elsewhere, as described below. Since the J2 V offset was so large, we assumed the electronics must be bad.

To sum, we found the following electronics suspect:

A18 V (offset due to electronics)

A18 H (electronics NOT tested, large sigma)

B12 H (offset due to electronics)

F12 H, V (sigma due to electronics, V not tested)

J02 V (offset due to electronics)

J14 V (electronics NOT tested, large sigma)

Something we did not realize until later, but is quite important, is that any electronics test, tests the electronics and everything downstream. Hence, the electronics could actually be fine, with the problem appearing in equipment in Bldg 929 or beyond. 
Notes:

Here we mention some items that didn't fit elsewhere. We had initially started using 5 AGS magnet cycles for our averages and sigmas. I became worried that a single bad cycle could throw off our sigma measurement, in other words the sigma could vary from one five cycle measurement to the next. We did a trial at L2 with only a $40 \mathrm{mV}$ signal (smaller than the $380 \mathrm{mV}$ we normally used) and received the following sigmas: $4.1,2.6,1.7,1.9,2.5,1.6,2.1,2.3,2.3,2.1,1.8$, and 1.7 , over 5 cycles. The variation here, especially the first of 4.1, led me to think we should look at more cycles. After this, $10 / 26$ and after, we used 15 cycles.

On 10/31 in the afternoon, we started receiving the message "Done clearing board errors" from AgsOrbitDisplay. The program would show no data when this message came up. After some erratic results, we stopped getting data at all. A call to the programmer, Seth Nemesure, and then to the Controls group, Brian Oerter, led to the knowledge that a scalar board was malfunctioning. This was replaced that evening and the next day we could record data again.

After this, we noticed that F14 had a anomalous sigma in the horizontal (about $200 \mathrm{~mm}$ ). Several days later we checked and F14 H and F4 H now had large offsets. Since this contrasted with our recent tickle tests, we asked Brian Oerter to investigate. It was determined that the board that had been replaced on 10/31 was defective as well. Another board was installed and the data looked good. This is the important information alluded to above, that boards in Bldg 929 can cause both large offsets and sigmas. Any future testing methods should take this into account. Please see references at end.

We have mentioned that the electronics needs the LO signal to work properly. On 11/1 the RF group had to remove this signal. To complete our tests, we had to generate an approximation to this signal. One of us, RS, used a dual channel frequency generator (Tektronix AFG 3102) to send a sine wave to both the electronics and electrodes, with frequencies 29.9 MHz and 8.5 MHz, respectively. Unlike the real LO used prior, this frequency did not sweep, causing it to be unnecessary to take data at all 14 times. We did nonetheless, just for sake of comparison. With the real LO there was a difference on any BPM during the 14 times (see Figure 3); with the synthetic LO there was little difference.

During testing RS noticed that many of the units used to keep the electronics cool were broken or missing fans. In the interest of keeping the electronics from malfunctioning, these were fixed as quickly as possible.

\section{The Decisions:}

The physicist was given a table representing the testing data. From this he created a list of recommendations of how to switch around electronics and matchboxes so that the most important superperiods in the ring had a complete set of working BPM's. The following decisions were made:

* Remove electronics from H14, I4, and K4

* Replace the electronics in A18, B8, C4, and F12 
* Remove matchboxes from $\mathrm{H} 14, \mathrm{I} 4$, and $\mathrm{K} 4$ and replace with 1 ns jumpers.

* Replace matchboxes at E4, F2, and J2

\section{Things Changed:}

We carried out the recommendations above. We moved the $\mathrm{H} 14$ electronics to A18, I4 to B8 (had no electronics), K4 to C4 (had no electronics), and C12 (from the shop) to F12. The old electronics from A18 and F12 were taken back to the shop. We put the K4 matchbox at J2 (which had bad connectors and was removed) and the I4 matchbox at E4 (where we had thought the phase looked wrong on one channel). The H14 matchbox was NOT removed because it was in a strange physical orientation. In place of the removed matchboxes were installed 1 ns jumpers.

After these changes we performed tickle tests on the moved equipment to ensure it was in working order. For C4 and F12 we saw great data, but for B8 we had sigmas around 1.5. This is still below our cut-off of 1.8, but a little high. We will have to see how it reacts to beam. Before we took the electronics from K4, but after we had removed the matchbox, we tested it. We saw a large offset in the horizontal that we could not correct. Perhaps this was caused by the removal of the matchbox.

Testing A18 with the new electronics led to disappointment. The offset in the vertical was still there. Now, instead of being stuck on a reading of -450 , it was stuck on a reading of -37 . No matter how it was connected we saw this reading. RS had brought along a fiber optic function generator especially designed to model the electronics output. Using this and what we assumed were the right ports on the fiber optic patch panel we still could not get the offset to move. From our experience with the scalar board replacement that had helped F4 and F14, we placed a call into the Controls group.

Bill Venegas and John Pepe responded, going to Bldg 929 to test from there. They reported no problems. With RS and John in the A10 house and Bill reading the schematic over the phone, it was determined that the fiber optic cables running from the electronics to the fiber optic patch panel had been misconnected. With the proper connections made, everything seemed to work fine. The intensity signal had been fed to the vertical channel, and the vertical had been fed to the horizontal. Perhaps this should have been caught during the first tickle test, but alas it was not.

This experience led RS to consider that perhaps the A18 electronics were fine all along. Having a good set he decided to place them at I4. This would allow us to have a working set of electronics at a location where the matchbox had been removed. Since during our testing we had raised doubts about whether the matchboxes worked correctly, there had been some talk of simply removing them all. The Booster BPM system worked fine with no matchboxes. It was a concern how the system would operate without matchboxes so it is necessary for us to answer that question during this run. The electronics at I4 should help in that endeavor. 
BPM by BPM breakdown (test results and changes):

If any of the above seems jumbled, here is a breakdown of what the tests showed and what equipment changes we made for every BPM. If a BPM is not listed, it tested okay. A bad offset is only mentioned if it was bad no matter how we made connections.

A02:

H: LA thought was bad, we found a large sigma not due to electronics

A08:

maybe a bad matchbox (decibel loss differences of $20 \mathrm{~dB}$ )

A18:

H: LA thought was bad, we found nothing

$\mathrm{H}$ : tested a bad sigma

V: LA thought was bad, tested a bad offset

moved $\mathrm{H} 14$ electronics here and connected fiber optics correctly.

moved old A18 electronics to I4

B04:

LA thought was bad $(\mathrm{H} \& \mathrm{~V})$, we found nothing.

B08:

Had no electronics. Moved I4 electronics here. Tested a sigma of about 1.5 both channels. See how it reacts to beam.

B12:

$\mathrm{H}$ : Bad offset and maybe sigma traced to electronics

$\mathrm{V}$ : LA thought was bad, we found bad offset not due to electronics

B18:

No electronics

C04:

No electronics, moved K4 electronics here. Tested okay.

C12:

No electronics, moved "C12" electronics found in shop to F12

E04:

H: LA thought was bad, we found nothing

V: LA thought was bad, we found nothing

F02:

We found a strange phase on the matchbox. Moved I4 matchbox to here.

V: LA thought was bad, we found nothing.

We found a strange phase on the matchbox, but did nothing to correct.

F08:

No electronics

F12:

H: LA thought was bad, we found a bad sigma due to electronics

$\mathrm{V}$ : LA thought was bad, we found a bad sigma due to electronics

The scalar board for this has been changed since the measurements, may have affected the sigmas. Nonetheless we moved the $\mathrm{C} 12$ electronics to here. Tested okay. 
F14:

V: LA thought was bad, we found a bad offset not due to electronics. Matchbox too close to radiation to test, though we tightened several very loose connections on it. Check for improvement.

G14:

No electronics

H02:

H14:

V: offset of 37 on two plates, not investigated further. Probably okay.

Took electronics from here for A18, left matchbox alone

I04:

Took electronics from here for B8. After this, moved A18 electronics here.

Moved matchbox from here to E4.

J02:

H: LA thought was bad, we found nothing

$\mathrm{V}$ : LA thought was bad, we found a large offset due to electronics

matchbox had bad connectors, moved K4 matchbox here

J14:

V: LA thought was bad, we found bad sigma and disconnected wire inside matchbox. Re-soldered but did not re-test.

K02:

V: had a high sigma at just one time (out of 14). Probably okay.

K04:

Took electronics from here to C4. Moved matchbox to J2. Tested with electronics but no matchbox and found a bad horizontal offset.

K18:

H: LA thought was bad, we found nothing

Many intensities were below normal, though we have decided to ignore this.

Results of the Changes (with beam 2007):

When beam was restored in the AGS for the 2007 run we were able to check whether our changes had made a difference, and also if BPM's which passed our test were truly good. The changes and before and after conditions of the BPM's are summarized in Appendix VII. Five channels (either H or V) are good this year, despite no changes having been made. As the run progresses, these may yet evidence faults. Ten channels are good this year, caused hopefully by our changes:

A18 V: Electronics were swapped and cabling was corrected

B8 H \& V: Electronics were added

C4 H \& V: Electronics were added

E4 V: Swap of matchbox seems effective

J2 H: Swap of matchbox seems effective

$\mathrm{J} 14 \mathrm{~V}$ : matchbox repaired

The bulk of BPM's brought back on line is due to the addition or replacement of electronics. We presently do not have enough electronics for all locations, and it is 
imperative that more are built to fill these locations and replace anymore that fail. The changing of matchboxes seems to be effective and warrants further investigation. The one location where the matchbox was removed, I4, now reports one bad channel whereas before both worked. Whether all the matchboxes can (or should) be removed from the ring is still an open question.

We lost H14 and K4 this year due to the use of their electronics elsewhere at more vital locations. While the replaced matchbox seemed to help $\mathrm{J} 2 \mathrm{H}$, the vertical channel is still bad and the electronics are suspected. Both channels of F14 are now bad, though our testing had revealed a problem (large offset) but did not lead to a cause (the electronics were deemed okay). The E4 H channel was not improved by the matchbox swap, and our tickle test detected nothing. For B12, testing showed possible electronics malfunction but no corrective steps were taken. Presently neither channel functions properly.

F12, which had a chassis replacement, was good for most of the run but developed problems near the end. The chassis moved here was something found in the shop marked C12. The history of this chassis is not known in detail. Also of interest is that K18 $\mathrm{H}$ worked fine for the first couple weeks of the run before going bad again. This one was bad last year, but we found nothing with our tests and made no changes. The reason for it going in and out of operation is not known. Others which "spontaneously" went from bad to good with no apparent help from us and stayed that way are: A2 H, A8 H, B4 H \& V, and F2 V. Possible explanations here include the tightening and remaking of connections or other aspects of the system not investigated.

In conclusion, while our testing method may need some refinements for future use, it was successful in identifying some problem BPM's without beam in the machine and pointing us to possible solutions. Some BPM's, which are bad with beam, were not detected by our tests. This is a concern. The issue of whether matchboxes are vital to system operation and how to diagnose them is unresolved. The greatest lesson to be gleaned is that the electronics - either matchboxes, scalar boards, or chassis-are vital to the system and perhaps the weakest link. Spares of all devices should be obtained and their testing procedures refined.

Special thanks are due to Tom Russo, Justin Gullotta, Tony Curcio, and Kevin Smith for their kindness in reviewing this paper and making many vibrant, vital comments. It goes without saying that any remaining errors are mine alone (MPM). 
Those flagged by sigma $=1.8$ :

\section{Appendix I}

$\begin{array}{lcccc}\text { BadBPM } & \text { Sigma } & \text { Offset } & \text { Intensity } & \text { Time } \\ \text { H_A02 } & 2.054 & -32.300 & 8580.800 & 200 \\ \text { H_A02 } & 3.322 & -20.321 & 8541.730 & 300 \\ \text { H_A02 } & 2.461 & -16.711 & 8538.530 & 350 \\ \text { H_A02 } & 2.771 & -15.850 & 8536.530 & 400 \\ \text { H_A02 } & 2.731 & -15.600 & 8539.070 & 450 \\ \text { H_A02 } & 2.663 & -14.586 & 8536.270 & 500 \\ \text { H_A02 } & 3.144 & -15.028 & 8534.400 & 550 \\ \text { H_A02 } & 2.178 & -14.059 & 8534.670 & 600 \\ \text { H_A02 } & 2.487 & -14.920 & 8534.130 & 650 \\ \text { H_A18 } & 2.749 & -3.763 & 8542.000 & 50 \\ \text { H_A18 } & 2.107 & -3.009 & 8543.870 & 100 \\ \text { H_A18 } & 2.592 & -4.216 & 8541.730 & 150 \\ \text { H_A18 } & 2.330 & -5.621 & 8548.130 & 200 \\ \text { H_B12 } & 1.971 & 41.440 & 8602.930 & 50 \\ \text { H_B12 } & 2.521 & 41.191 & 8601.330 & 100 \\ \text { H_B12 } & 2.836 & 40.845 & 8602.400 & 150 \\ \text { H_B12 } & 2.237 & 41.112 & 8604.000 & 200 \\ \text { H_B12 } & 2.296 & 42.115 & 8604.270 & 250 \\ \text { H_B12 } & 3.230 & 41.072 & 8598.800 & 300 \\ \text { H_B12 } & 2.210 & 40.381 & 8602.130 & 350\end{array}$

Those flagged by absolute offset $=30$ :

$\begin{array}{lcccc}\text { H_B12 } & 2.583 & 41.440 & 8605.600 & 400 \\ \text { H_B12 } & 2.422 & 42.075 & 8601.070 & 450 \\ \text { H_B12 } & 2.593 & 40.851 & 8597.470 & 500 \\ \text { H_B12 } & 2.431 & 39.746 & 8603.330 & 550 \\ \text { H_B12 } & 2.510 & 41.412 & 8605.470 & 600 \\ \text { H_B12 } & 2.331 & 41.843 & 8599.600 & 650 \\ \text { H_B12 } & 2.127 & 40.488 & 8599.200 & 700 \\ \text { H_F12 } & 2.245 & 47.861 & 8626.400 & 150 \\ \text { H_F12 } & 1.924 & 49.651 & 8600.670 & 300 \\ \text { H_F12 } & 2.180 & 47.900 & 8598.000 & 400 \\ \text { H_F12 } & 1.883 & 47.742 & 8598.670 & 450 \\ \text { H_F12 } & 1.902 & 46.931 & 8598.400 & 600 \\ \text { H_F12 } & 2.013 & 48.592 & 8599.330 & 650 \\ \text { H_F12 } & 2.050 & 48.036 & 8596.400 & 700 \\ \text { V_F12 } & 6.205 & 5.987 & 8627.070 & 50 \\ \text { V_F12 } & 6.450 & 2.727 & 8629.870 & 100 \\ \text { V_F12 } & 6.147 & -0.000 & 8632.530 & 150 \\ \text { V_F12 } & 8.288 & 0.240 & 8641.730 & 200 \\ \text { V_F12 } & 5.695 & -6.693 & 8629.730 & 250 \\ \text { V_F12 } & 7.423 & -5.533 & 8607.330 & 300 \\ \text { V_F12 } & 6.753 & -3.960 & 8608.930 & 350\end{array}$

Offset cutoff value: 30.000

BadBPM Offset Sigma Intensity Time

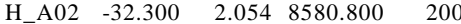

$\begin{array}{lllll}\text { H_A02 } & -31.484 & 1.720 & 8557.470 & 250\end{array}$

$\begin{array}{lllll}\text { V_A02 } & -62.380 & 0.829 & 8573.330 & 50\end{array}$

$\begin{array}{lllll}\text { V_A02 } & -62.347 & 0.965 & 8573.730 & 100\end{array}$

$\begin{array}{lllll}\mathrm{V} \_\mathrm{A} 02 & -62.953 & 1.120 & 8575.200 & 150\end{array}$

$\begin{array}{lllll}\text { V_A02 } & -63.393 & 1.110 & 8555.070 & 200\end{array}$

$\begin{array}{lllll}\text { V_A02 } & -66.720 & 0.892 & 8545.200 & 250\end{array}$

$\begin{array}{lllll}V_{-} A 02 & -66.640 & 1.138 & 8546.270 & 300\end{array}$

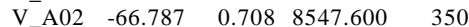

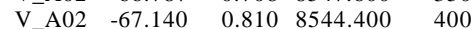

$\begin{array}{lllll}\text { V_A02 } & -66.627 & 0.947 & 8548.400 & 450\end{array}$

$\begin{array}{lllll}V_{-} A 02 & -66.720 & 1.108 & 8545.070 & 500\end{array}$

$\begin{array}{lllll}\text { V_A02 } & -67.193 & 0.833 & 8545.870 & 550\end{array}$

$\begin{array}{lllll}\text { V_A02 } & -66.940 & 0.840 & 8546.130 & 600\end{array}$

$\begin{array}{lllll}\text { V_A02 } & -67.187 & 1.065 & 8546.400 & 650\end{array}$

$\begin{array}{lllll}\text { V_A02 } & -66.753 & 0.985 & 8549.730 & 700\end{array}$

$\begin{array}{lllll}\text { V_A18 } & -450.000 & 0.000 & 8550.270 & 50\end{array}$

$\begin{array}{lllll}\text { V_A18 } & -450.000 & 0.000 & 8553.730 & 100\end{array}$

$\begin{array}{lllll}\text { V_A18 } & -450.000 & 0.000 & 8553.070 & 150\end{array}$

$\begin{array}{lllll}\text { V_A18 } & -450.000 & 0.000 & 8557.730 & 200\end{array}$

$\begin{array}{lllll}\text { V_A18 } & -450.000 & 0.000 & 8580.000 & 250\end{array}$

$\begin{array}{lllll}\text { V_A18 } & -450.000 & 0.000 & 8593.600 & 300\end{array}$

$\begin{array}{lllll}\text { V_A18 } & -450.000 & 0.000 & 8597.070 & 350\end{array}$

$\begin{array}{lllll}\text { V_A18 } & -450.000 & 0.000 & 8597.870 & 400\end{array}$

$\begin{array}{lllll}\text { V_A18 } & -450.000 & 0.000 & 8598.800 & 450\end{array}$

$\begin{array}{lllll}\text { V_A18 } & -450.000 & 0.000 & 8599.200 & 500\end{array}$

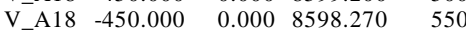

$\begin{array}{lllll}\mathrm{V}_{-} \mathrm{A} 18 & -450.000 & 0.000 & 8598.130 & 600\end{array}$

$\begin{array}{lllll}\text { V_A18 } & -450.000 & 0.000 & 8599.470 & 650\end{array}$

$\begin{array}{lllll}\text { V_A18 } & -450.000 & 0.000 & 8598.800 & 700\end{array}$

$\begin{array}{lllll}\text { H_B12 } & 41.440 & 1.971 & 8602.930 & 50\end{array}$

$\begin{array}{lllll}\text { H_B12 } & 41.191 & 2.521 & 8601.330 & 100\end{array}$

$\begin{array}{lllll}\text { H_B12 } & 40.845 & 2.836 & 8602.400 & 150\end{array}$

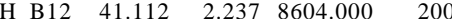

$\begin{array}{lllll}\text { H B } 12 & 42.115 & 2.296 & 8604.270 & 250\end{array}$

H_B12 $41.072 \quad 3.230 \quad 8598.800 \quad 300$

$\begin{array}{lllll}\text { H_B12 } & 40.381 & 2.210 & 8602.130 & 350\end{array}$

$\begin{array}{lllll}\text { H_B12 } & 41.440 & 2.583 & 8605.600 & 400\end{array}$

$\begin{array}{lllll}\text { H_B12 } & 42.075 & 2.422 & 8601.070 & 450\end{array}$

$\begin{array}{lllll}\text { H_B12 } & 40.851 & 2.593 & 8597.470 & 500\end{array}$

$\begin{array}{lllll}\text { H_B12 } & 39.746 & 2.431 & 8603.330 & 550\end{array}$

$\begin{array}{lllll}\text { H_B12 } & 41.412 & 2.510 & 8605.470 & 600\end{array}$

$\begin{array}{lllll}\text { H_B12 } & 41.843 & 2.331 & 8599.600 & 650\end{array}$

$\begin{array}{lllll}\text { H_B12 } & 40.488 & 2.127 & 8599.200 & 700\end{array}$

$\begin{array}{lllll}\text { V_B12 } & 70.987 & 0.495 & 8636.800 & 50\end{array}$

$\begin{array}{ccccc}\text { V_B12 } & 70.487 & 0.908 & 8637.070 & 100\end{array}$ $\begin{array}{lllll}\text { V_B12 } & 71.240 & 0.740 & 8636.130 & 150\end{array}$

$\begin{array}{lllll}\text { V_B12 } & 70.960 & 0.900 & 8636.930 & 200 \\ \text { V_B12 } & 71.007 & 0.506 & 8636.800 & 250\end{array}$

$\begin{array}{lllll}\text { V_B12 } & 71.240 & 0.711 & 8635.870 & 300\end{array}$

$\begin{array}{lllll}\text { V_B12 } & 70.600 & 1.053 & 8637.600 & 350\end{array}$

$\begin{array}{lllll}\text { V_B12 } & 70.667 & 0.629 & 8636.930 & 400\end{array}$

$\begin{array}{lllll}\text { V_B12 } & 70.927 & 0.559 & 8637.070 & 450\end{array}$

$\begin{array}{lllll}\text { V_B12 } & 70.907 & 0.803 & 8636.670 & 500\end{array}$

$\begin{array}{lllll}\text { V_B12 } & 70.973 & 0.540 & 8636.930 & 550\end{array}$

$\begin{array}{lllll}\text { V_B12 } & 71.180 & 0.852 & 8636.000 & 600\end{array}$

$\begin{array}{lllll}\mathrm{V} B \mathrm{~B} 12 & 71.133 & 0.682 & 8635.730 & 650\end{array}$

$\begin{array}{lllll}\mathrm{V} \_\mathrm{B} 12 & 71.147 & 0.781 & 8637.730 & 700\end{array}$

H_F12 $48.592 \quad 1.364 \quad 8627.730 \quad 50$

$\begin{array}{lllll}\text { H_F12 } & 48.835 & 1.217 & 8626.930 & 100\end{array}$

$\begin{array}{lllll}\text { H_F12 } & 47.861 & 2.245 & 8626.400 & 150\end{array}$

$\begin{array}{lllll}\text { H_F12 } & 52.275 & 0.930 & 8646.800 & 200\end{array}$

$\begin{array}{lllll}\text { H_F12 } & 52.609 & 1.214 & 8616.270 & 250\end{array}$

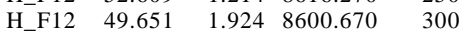

H_F12 $47.600 \quad 1.501 \quad 8597.330 \quad 350$

$\begin{array}{lllll}\text { H_F12 } & 47.900 & 2.180 & 8598.000 & 400\end{array}$

$\begin{array}{lllll}\text { H_F12 } & 47.742 & 1.883 & 8598.670 & 450\end{array}$

H_F12 $47.362 \quad 1.203 \quad 8597.070 \quad 500$

H_F12 $48.286 \quad 1.659 \quad 8599.730 \quad 550$

H_F12 $46.931 \quad 1.902 \quad 8598.400 \quad 600$

$\begin{array}{lllll}\text { H_F12 } & 48.592 & 2.013 & 8599.330 & 650\end{array}$

$\begin{array}{lllll}\text { H_F12 } & 48.036 & 2.050 & 8596.400 & 700\end{array}$

$\begin{array}{lllll}\text { H_F14 } & 33.450 & 0.602 & 8638.000 & 250\end{array}$

$\begin{array}{lllll}\text { H_F14 } & 30.923 & 0.837 & 8633.730 & 300\end{array}$

$\begin{array}{lllll}\text { H_F14 } & 30.283 & 0.761 & 8630.670 & 350\end{array}$

$\begin{array}{lllll}\text { H_F14 } & 30.062 & 0.982 & 8629.070 & 400\end{array}$

H_F14 $30.130 \quad 0.883 \quad 8627.200 \quad 550$

$\begin{array}{lllll}\text { V_F14 } & 65.493 & 0.311 & 8629.470 & 50\end{array}$

$\begin{array}{lllll}\text { V_F14 } & 65.553 & 0.309 & 8628.670 & 100\end{array}$

$\begin{array}{lllll}\text { V_F14 } & 65.367 & 0.383 & 8630.400 & 150\end{array}$

$\begin{array}{lllll}\text { V_F14 } & 64.933 & 0.559 & 8593.870 & 200\end{array}$

$\begin{array}{lllll}\text { V_F14 } & 63.960 & 0.818 & 8582.530 & 250\end{array}$

$\begin{array}{lllll}\text { V_F14 } & 63.593 & 0.578 & 8616.000 & 300\end{array}$

$\begin{array}{lllll}\text { V_F14 } & 63.067 & 0.676 & 8620.670 & 350\end{array}$

$\begin{array}{lllll}\text { V_F14 } & 62.960 & 0.710 & 8622.400 & 400\end{array}$

$\begin{array}{lllll}\text { V_F14 } & 63.253 & 0.561 & 8620.670 & 450\end{array}$

$\begin{array}{lllll}\text { V F F14 } & 63.093 & 0.439 & 8621.070 & 500\end{array}$

$\begin{array}{lllll}\text { V_F14 } & 63.027 & 0.530 & 8620.670 & 550\end{array}$

$\begin{array}{lllll}\text { V_F14 } & 62.767 & 0.807 & 8622.400 & 600\end{array}$

$\begin{array}{lllll}\text { V_F14 } & 62.900 & 0.588 & 8622.670 & 650\end{array}$

$\begin{array}{lllll}\text { V_F14 } & 63.120 & 0.453 & 8621.470 & 700\end{array}$

$\begin{array}{lllll}\text { V_H02 } & 37.720 & 0.452 & 8735.070 & 50\end{array}$

$\begin{array}{lllll}\text { V_H02 } & 37.693 & 0.580 & 8734.270 & 100\end{array}$

$\begin{array}{lllll}\text { V_H02 } & 37.127 & 0.613 & 8735.070 & 150\end{array}$ 


\section{Appendix II}

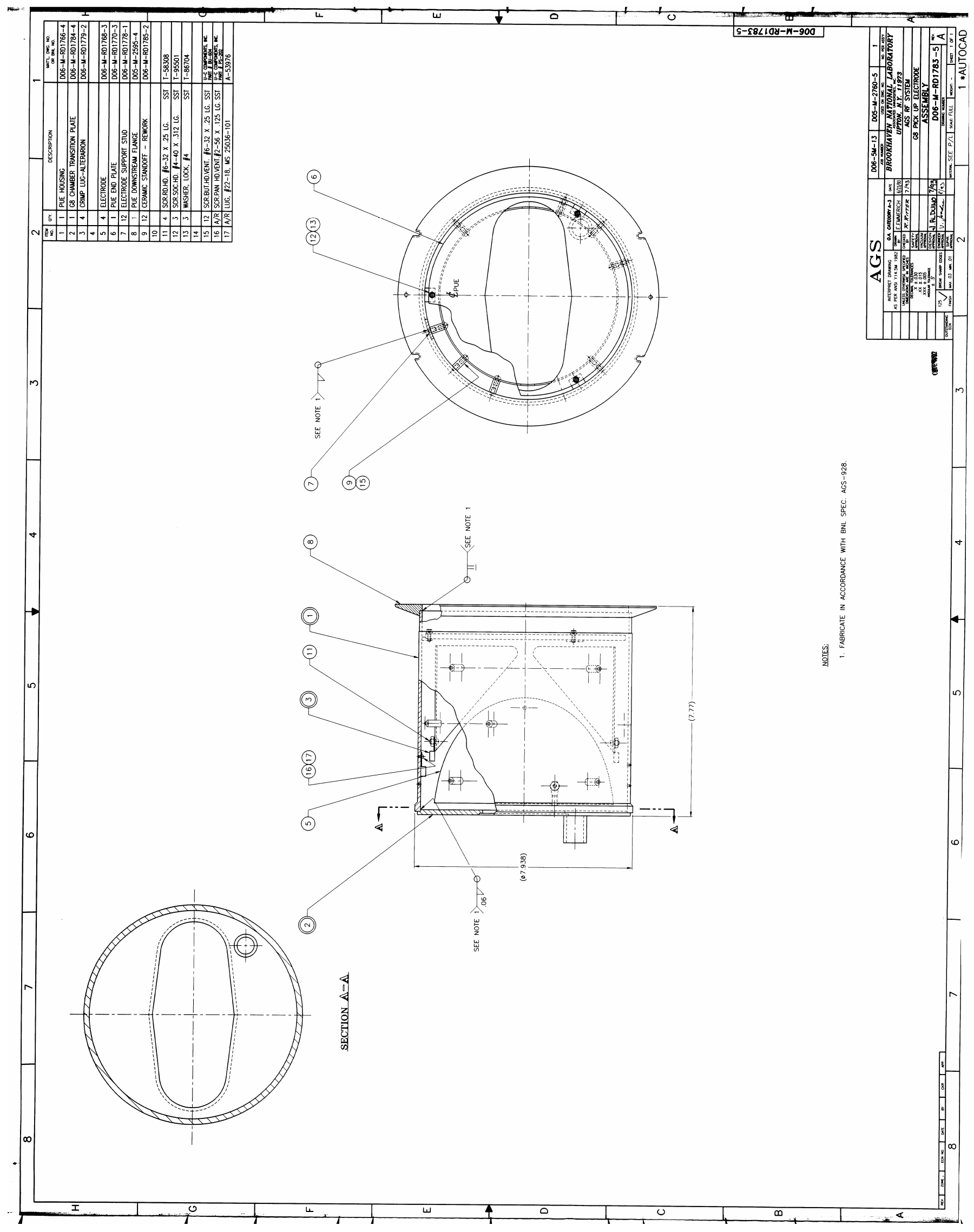




\section{Appendix III}

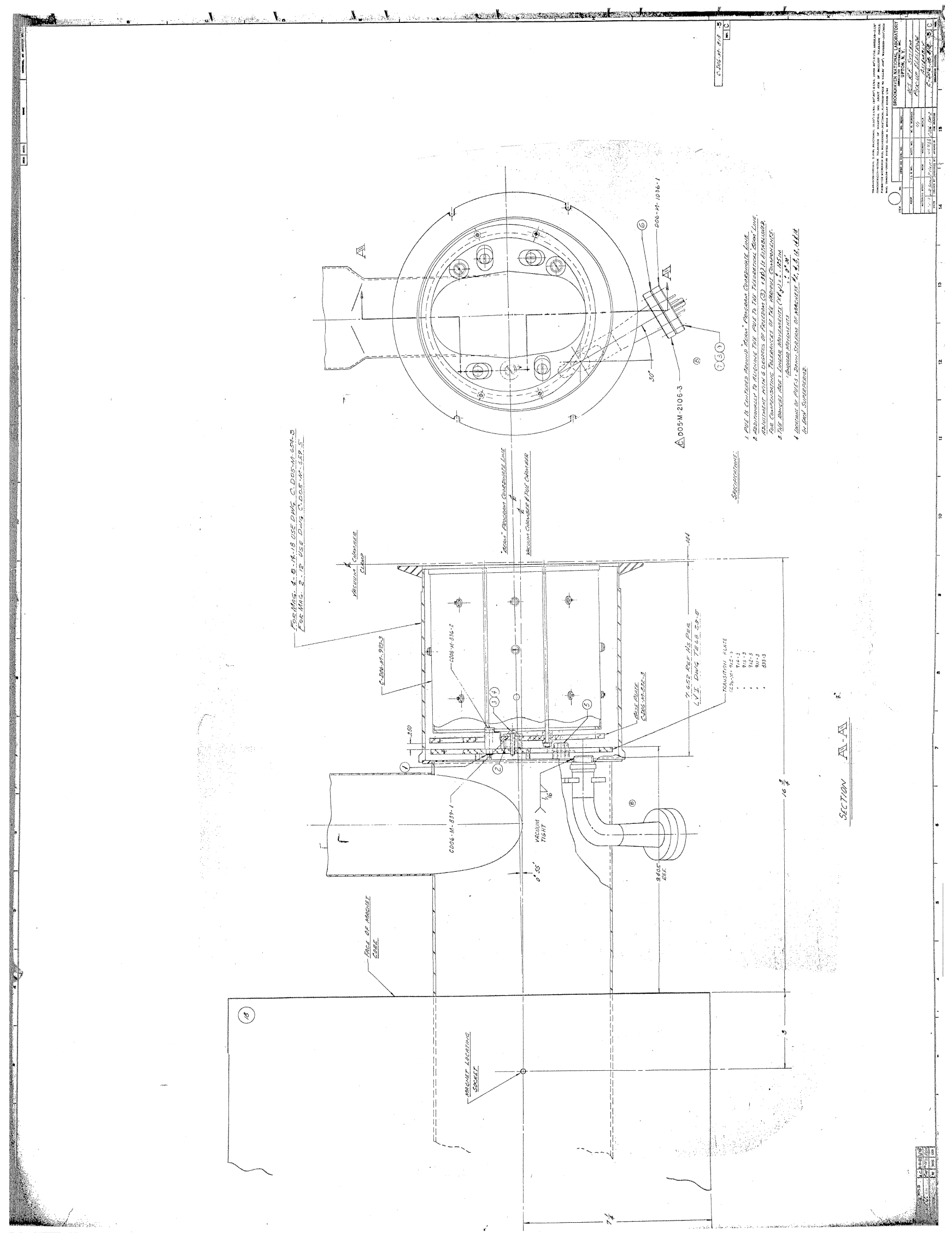




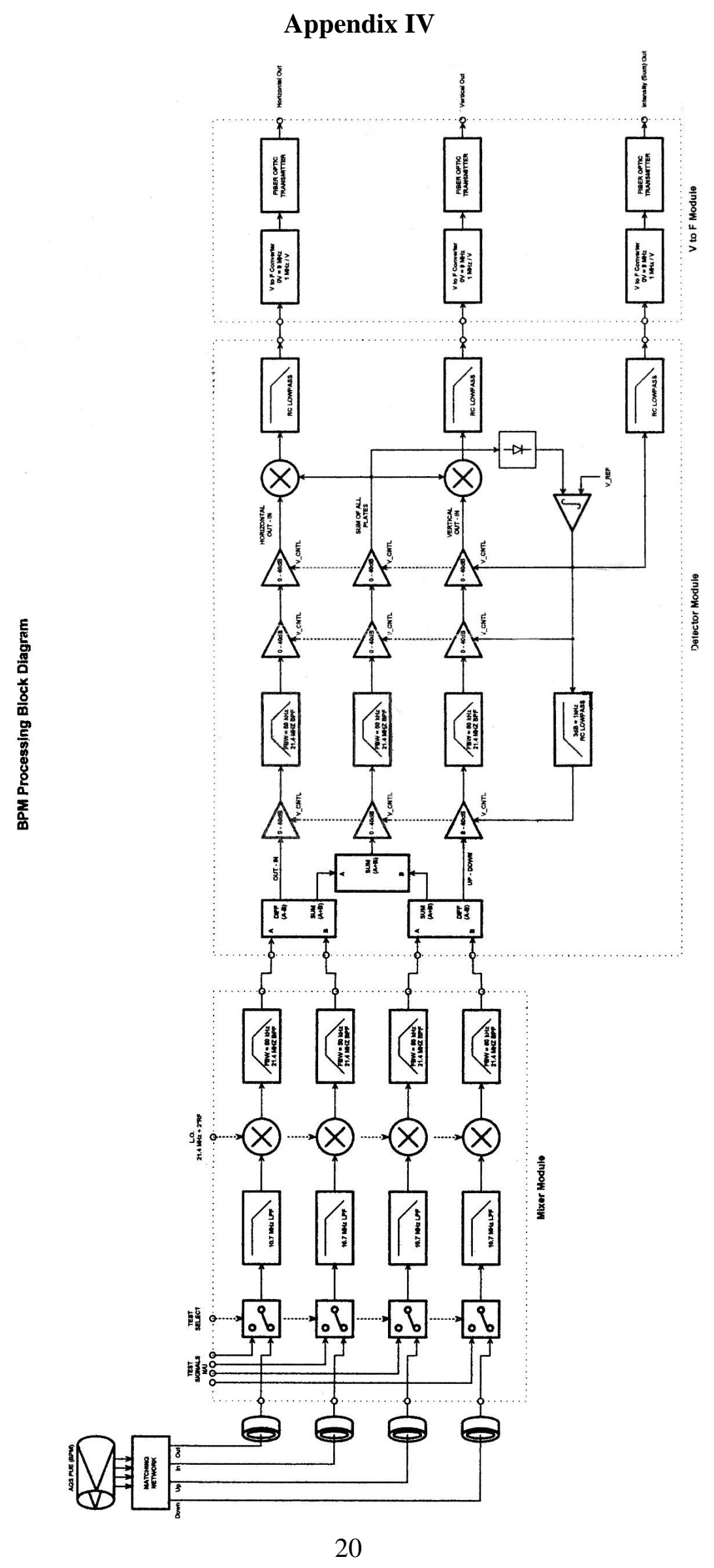




\section{Appendix V}

Kevin Smith

$10 / 09 / 2003$

\section{AGS \& Booster BPM System Random Bits of Information}

1) The source of the BPM (PUE) signals is current induced in the PUE plates as a bunch passes by. The coupling in the beam-PUE system is fundamentally a high pass filter type of response. No DC signal is coupled, therefore the system will only "see" bunched beam, or any RF "structure" on a DC coasting beam if the amplitude of this structure is high enough.

2) Because beam bunches are the source of the signals in the BPM system, the spectrum of these bunches is dominated by harmonics of the beam revolution frequency (the frequency at which a bunch circulates in the machine). Betatron and synchrotron motion will also produce lines in the spectrum.

3) In an idealized case where the RF system is running harmonic number " $h$ " (harmonic number always refers to a harmonic of the beam revolution frequency) at frequency $\mathrm{h} *$ frev, there will be "h" equally populated, equally spaced bunches circulating in the ring. Because they are identical, an observer (PUE) cannot tell which is which and therefore has no kno wledge of the revolution frequency. Thus the spectrum of the PUE signal will contain "lines" at frequency $\mathrm{h} *$ frev (the RF fundamental), $2 * \mathrm{~h} *$ frev (the $2^{\text {nd }}$ harmonic of the RF), etc..

4) If you look at the PUE signal broadband (i.e. all significant harmonics are included) on a scope, you see something that has the shape of the bunch in time. If you filter the PUE signal and select only a particular harmonic of interest, you see a sine wave with a certain amplitude and phase. The amplitude of that sine wave depends on the beam position relative to a PUE plate, the beam intensity (current) and the shape of the bunch. The BPM processing electronics are only interested in narrowband $(\sim 20 \mathrm{kHz} \mathrm{BW})$ processing of a specific spectral component - the $2^{\text {nd }}$ harmonic of the RF.

5) The BPM processing electronics are designed to cover a very large dynamic range $(\sim 100 \mathrm{~dB})$. This dynamic range comes about primarily from two effects mentioned in (4): I) Directly as a result of variations in beam intensity (high intensity protons vs. low intensity heavy ions), and II) Bunch shape effects on the amplitude of the sprectral components. The useable dynamic range depends on how much the user is willing to average.

6) The PUEs are ideally constructed such that each plate (outside, inside, up or down) produces a signal with an amplitude which varies linearly as the centroid of the beam moves toward or away from it. Ideally, plates in a given plane (horizontal or vertical) are not sensitive to the motion of the beam in the opposite plane.

7) PUEs are normally located coincident with machine main focusing quadrupoles (the locations of the machine beta maxes). In a machine like Booster, there are distinct 
horizontal and vertical main focusing quadrupoles, and there are horizontal and vertical PUEs at most of these locations. Even numbered locations (A4, B6) are horizontal PUEs and odd numbered locations are vertical. In AGS, a combined function machine, the focusing quadrupoles are "part" of the main dipoles (the pole faces are shaped appropriately to produce a quadrupole component in the magnetic field). PUEs in the AGS are combined horizontal and vertical pickups, located at points of "average" beta around the ring. There are six PUEs in each superperiod, at the downstream end of magnets $2,4,8,12,14$, and 18 .

7') (laa) A length scale for transverse position sampling is the length of a betatron oscillation. In Booster this is $\sim(6$ superperiods/ 4.8 oscillations $)=1.25$ superperiods. In AGS this is $\sim(12 \mathrm{sp} / 9 \mathrm{osc})=1.33$ superperiods. In a given plane a fully populated Booster would sample this length 24/4.8 $=5$ times and AGS 72/9 $=8$ times. Five samples per oscillation is just sufficient. The AGS measures at points of average beta ( less sensitivity ) but is able to tolerate many more missing BPM locations and still properly measure the dominant harmonics (near the betatron oscillation wavelength).

8) Because Booster BPMs are located next to main focusing quadrupoles, they are nominally located on the canonical "central orbit" of the machine, meaning a beam which is "centered" in the machine should induce equal signals on the PUE plates and produce a "zero" signal from the BPM electronics. This is not true for the AGS.

9) In both AGS and Booster, it was desired that the processing electronics be located outside the ring in service buildings. Therefore, in both systems, $1 / 4$ " heliax (solid shield flexible $50 \mathrm{ohm}$ coax) is used to bring signals from the PUEs in the ring to the service buildings. The signal runs can be as short as 100' and as long as 600'.

10) The only real topological differences between the AGS and Booster BPM signal runs are that:

A) The AGS system employs a matching network inserted between the PUE and the heliax in the ring. The main purpose of this network is to try and present a relatively high impedance to the PUE plates to flatten the frequency response. Booster employs a direct connection to the PUE.

B) Booster PUEs have an extra signal connection called the "cal ring". This is a ring which encircles the PUE plates and can be used to induce a test signal on the PUE plates. AGS PUEs have no cal ring.

C) Because AGS PUEs have four plates (two each for horizontal and vertical planes), there are four signal cables running between each PUE and a service building. Booster PUEs are single plane (horizontal or vertical), but because they include a cal ring, there are three signal cables running between each PUE and a service building. Also, there are two cal ring connections on each PUE - one is used for the signal cable, the other is terminated with a $1 \mathrm{w} 50 \mathrm{ohm}$ load.

11) All signal cables in a given PUE set are phase matched (i.e. matched in "electrical" length) so that RF signals from each plate, which are born phase coherent, remain so 
when they reach the BPM electronics. Phase of signals from one PUE to the next, or between the PUE RF signals and the BPM "L.O." signal are irrelevant.

12) It is important that all signal cables in a given PUE set also be amplitude matched, but it would be cost prohibitive to achieve this using external attenuators, and difficult (but possible) to achieve this by tweaking the BPM signal channel gains, so it is not attempted. Fortunately, test data taken during the AGS system installation showed typical amplitude matches across four cable sets on the order of $0.1 \mathrm{~dB}$. We'll discuss the effect of matching errors during the talk.

13) The only ground reference for the BPM electronics, cables, etc. (anything common to a heliax shield) is the machine beam pipe itself. The BPM chasses have provision for establishing a local ground, but this should not be used during normal machine running. The overall design philosophy of the system is to try and maintain a closed "faraday cage" along the entire signal chain.

14) The connection between the BPM system and the rest of the world is isolated via fiber optics. Analog signals representing beam position are converted to frequency encoded signals ( $\mathrm{V}$ to $\mathrm{F}$ converter), converted to optical and shipped to controls hardware on fiber. This brings up the other major difference between AGS and Booster BPM systems.

A) AGS analog signals (in the BPM chassis) are $+/-1 \mathrm{~V}=+/$ - full aperture. Fiber optic signals are encoded such that analog $0 \mathrm{~V}=9.0 \mathrm{MHz}=$ center aperture, and analog +/- $1 \mathrm{~V}=+/-1 \mathrm{MHz}=+/$ - full aperture.

B) Booster analog signals (in the BPM chassis) are $+/-1 \mathrm{~V}=+/$ - fill aperture. Booster fiber optic signals are encoded such that $30.0 \mathrm{MHz}=$ center aperture, and $+/-10 \mathrm{MHz}=+/$ - full aperture.

The internal hardware of the AGS and Booster V to F converter modules is completely different, but the physical packages are identical in order that the AGS V to F converters can be replaced with Booster style converters.

The intensity or sum signals are also encoded the same way, but the meaning is different than the position signals:

A) AGS and Booster analog intensity signals are $-0.6 \mathrm{~V}=$ min intensity, $+0.6 \mathrm{~V}=$ max intensity, $\mathrm{DV}=0.2 \mathrm{~V}=$ factor of ten in intensity (so the signal is logarithmic in intensity). Fiber optic signals are encoded such that analog $0 \mathrm{~V}=$ mid intensity $=9 \mathrm{MHz}$, and analog $+/-0.6 \mathrm{~V}=+/-0.6 \mathrm{MHz}=\max / \mathrm{min}$ intensity.

15) There are two basic ways of looking at BPM signals in MCR.

A) OrbitDisplay: Application reads scalars for each BPM. The scalars count the frequency encoded signal for over "gate width" set by the user. These counts are available as raw data, and translated into position in $\mathrm{mm}$. OrbitDisplay includes correction tables that can compensate for known offsets (and gain errors?). 
B) MCR Analog Mux: Crossbar allows up to four BPM analog signals in any combination to be selected for display on a scope. These signals have the calibrations described in (14) above with one caveat. If a scope channel is set to $50 \mathrm{ohm}$ input impedance, the calibration is as described in (14). If the channel is in high impedance, the calibration differs by a factor of ten, i.e. $1 \mathrm{~V}$ becomes $10 \mathrm{~V}$.

16) Polarity conventions: Regardless of any careless statements made above, or of the actual installed configuration, what OrbitDisplay and the analog mux signals say, polarities are supposed to be like so:

A) Directions are as the beam sees them.

B) Beam to the outside "Out" or high "Up" is a positive displacement from center. "In" and "Down" are negative. "Out" is made positive simply because beam to the outside means the orbit radius is larger.

C) "Out" and "In" mean just that:

I) Booster: "Out" = beam left (beam circulates clockwise), "In" = beam right.

II) AGS: "Out" = beam right (beam circulates counter-clockwise), "In" = beam left. 


\section{Appendix VI}
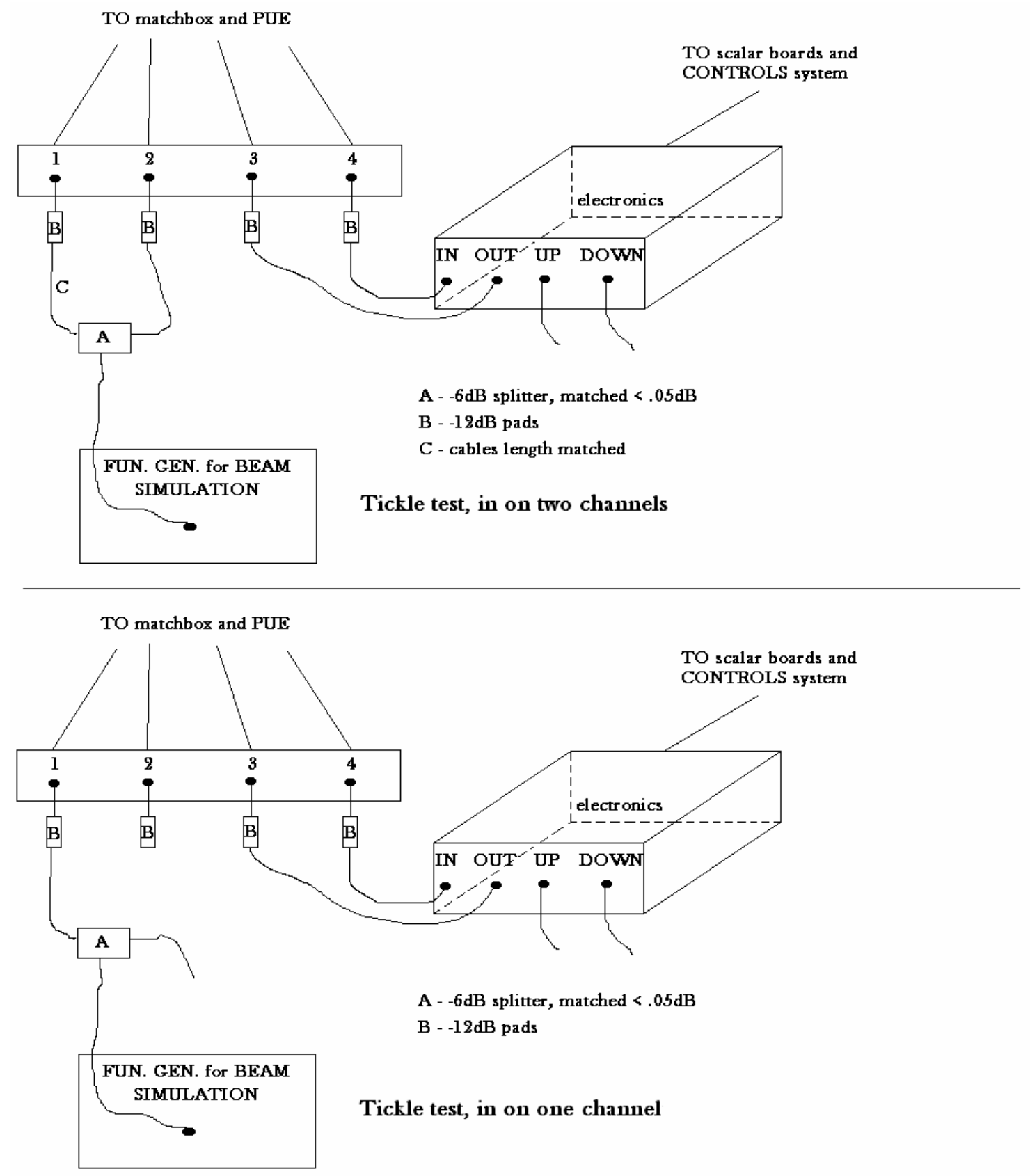

Tickle test, in on one channel 


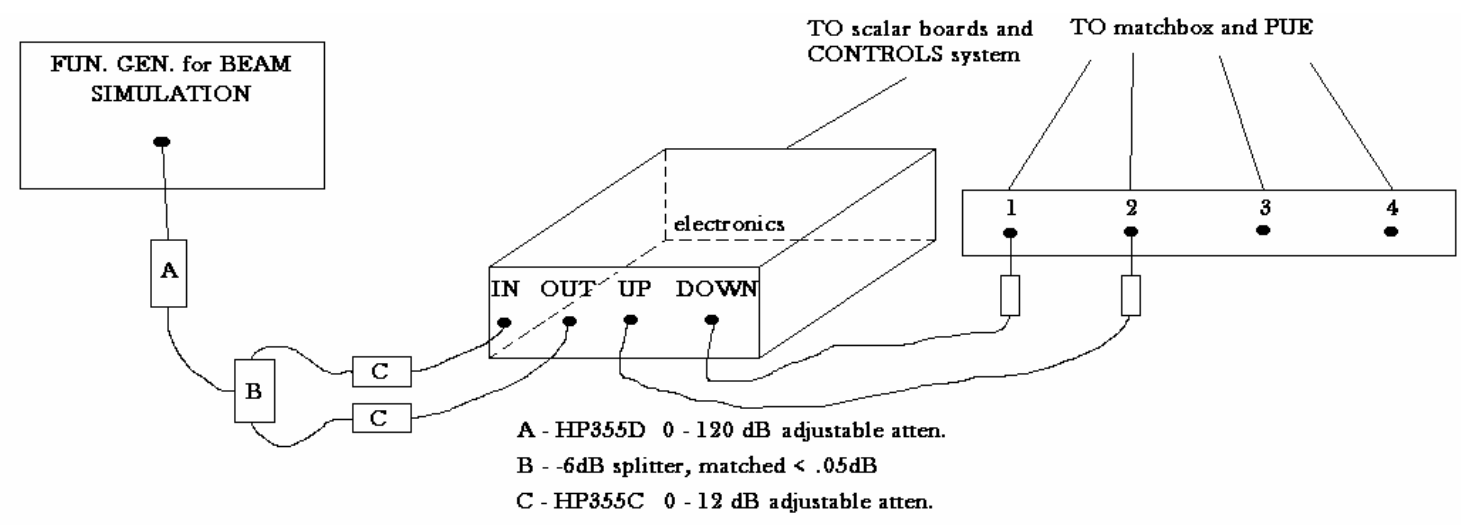

Electronics test

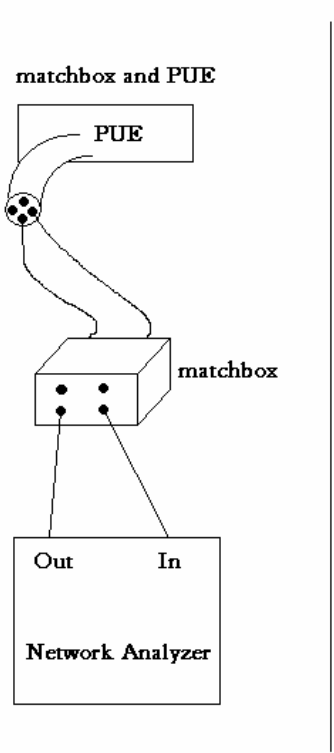

"Ring" tests

just matchbox

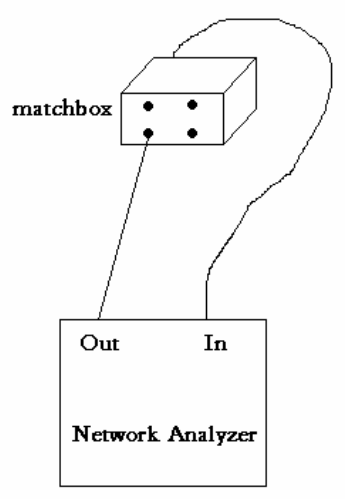

just PUE

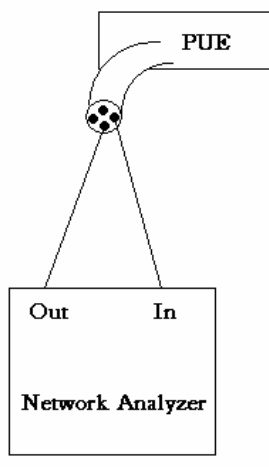




\section{Appendix VII (3/7/07)}

A1/A2/A3 = added $/$ removed $/$ replaced electronics B1/B2/B3/B4 $=$ added $/$ removed $/$ replaced $/$ repaired matchbox $\mathrm{C}=$ corrected wiring

\begin{tabular}{|c|c|c|c|c|c|c|c|c|c|c|c|c|}
\hline & A & A & A & A & A & A & A & A & A & A & A & A \\
\hline BPM & 2_H & 2_V & 4_H & 4_V & 8_H & 8_V & 12_H & 12_V & 14_H & 14_V & 18_H & 18_V \\
\hline Bad 2006 & X & & & & $x$ & & & & & & & $\mathrm{x}$ \\
\hline No Chassis '06 & & & & & & & & & & & & \\
\hline changes made & & & & & & & & & & & $A 3, C$ & $A 3, C$ \\
\hline Bad 2007 & & & & & & & & & & & & \\
\hline No Chassis '07 & & & & & & & & & & & & \\
\hline
\end{tabular}

\begin{tabular}{|c|c|c|c|c|c|c|c|c|c|c|c|c|}
\hline & $\mathrm{B}$ & B & B & B & B & $\mathrm{B}$ & B & B & B & B & B & B \\
\hline BPM & 2_H & 2_V & 4_H & 4_V & 8_H & 8_V & 12_H & 12_V & 14_H & 14_V & 18_H & 18_V \\
\hline Bad 2006 & & & $x$ & $x$ & & & & $x$ & & & & \\
\hline No Chassis '06 & & & & & $X$ & $x$ & & & & & $x$ & $x$ \\
\hline changes made & & & & & $\mathrm{A} 1$ & $\mathrm{~A} 1$ & & & & & & \\
\hline Bad 2007 & & & & & & & $X$ & $X$ & & & & \\
\hline No Chassis '07 & & & & & & & & & & & $x$ & $x$ \\
\hline
\end{tabular}

No Chassis '07

C

C $\quad$ C

C C

C $\quad \mathrm{C} \quad \mathrm{C}$

C

$\frac{\text { BPM }}{\text { Bad } 2006}$

No Chassis '06

changes made

Bad 2007

No Chassis '07

\begin{tabular}{|c|c|c|c|c|c|c|c|c|c|c|c} 
2_H & 2_V & 4_H & 4_V & 8_H & 8_V & 12_H & 12_V & 14_H & 14_V & 18_H & 18_V \\
\hline & & & & & & & & & & & \\
\hline & & $\mathrm{X}$ & $\mathrm{X}$ & & & $\mathrm{X}$ & $\mathrm{X}$ & & & & \\
\hline & & $\mathrm{A} 1$ & $\mathrm{~A} 1$ & & & & & & & & \\
\hline & & & & & & & & & & & \\
\hline
\end{tabular}

BPM

Bad 2006

No Chassis '06

changes made

Bad 2007

No Chassis '07

D D

D

D

D

D

$D$

D

D

D

D

\begin{tabular}{|l|l|}
\hline & \\
\hline & \\
\hline & \\
\hline
\end{tabular}

\section{BPM \\ Bad 2006}

No Chassis '06

changes made

Bad 2007

No Chassis '07

\section{BPM}

Bad 2006

No Chassis '06

changes made

Bad 2007

No Chassis '07

\begin{tabular}{|c|c|}
\hline 2_H & 2_V \\
\hline & \\
\hline & \\
\hline & \\
\hline & \\
\hline & E \\
\hline
\end{tabular}

4_H

\begin{abstract}
E E E E

E E E E

E

E E E E
\end{abstract}

4

\begin{tabular}{|c|c|c|c} 
4_H & 4_V & 8_H & 8_ \\
$\mathrm{X}$ & $\mathrm{X}$ & & \\
& & & \\
$\mathrm{B} 3$ & $\mathrm{~B} 3$ & & \\
$\mathrm{X}$ & & & \\
& & & \\
$\mathrm{F}$ & $\mathrm{F}$ & $\mathrm{F}$
\end{tabular}

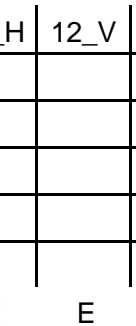

\begin{tabular}{|l|l|l|}
\hline & $14 \_H$ & 14 \\
\hline & & \\
\hline & & \\
\hline & & \\
\hline
\end{tabular}

\begin{tabular}{|l|l|l} 
14_V & 18_H & $18 \_\mathrm{V}$ \\
\hline & & \\
\hline & & \\
\hline & & \\
\hline & & \\
& &
\end{tabular}

\begin{tabular}{|c|c|c|c|c|c} 
12_H & 12_V & 14_H & 14_V & 18_H & $18 \_\mathrm{V}$ \\
\hline & & & & & \\
\hline & & & & & \\
\hline & & & & & \\
\hline & & & & & \\
\hline F & & & & & \\
\hline
\end{tabular}

\begin{tabular}{c|c|c|c|c|c|c|c|c|c|c|c|c|c}
$\mathrm{F}$ & $\mathrm{F}$ & $\mathrm{F}$ & $\mathrm{F}$ & $\mathrm{F}$ & $\mathrm{F}$ & $\mathrm{F}$ & $\mathrm{F}$ & $\mathrm{F}$ & $\mathrm{F}$ & $\mathrm{F}$ & $\mathrm{F}$ \\
2_H & 2_V & 4_H & 4_V & 8_H & 8_V & 12_H & 12_V & 14_H & 14_V & 18_H & 18_V \\
\hline & $\mathrm{X}$ & & & & & $\mathrm{X}$ & $\mathrm{X}$ & & $\mathrm{X}$ & & \\
\hline & & & & $\mathrm{X}$ & $\mathrm{X}$ & & & & & & \\
\hline & & & & & & $\mathrm{A} 3$ & $\mathrm{~A} 3$ & & & & \\
\hline & & & & & & & & $\mathrm{X}$ & $\mathrm{X}$ & & \\
\hline & & & & $\mathrm{X}$ & $\mathrm{X}$ & & & & & &
\end{tabular}

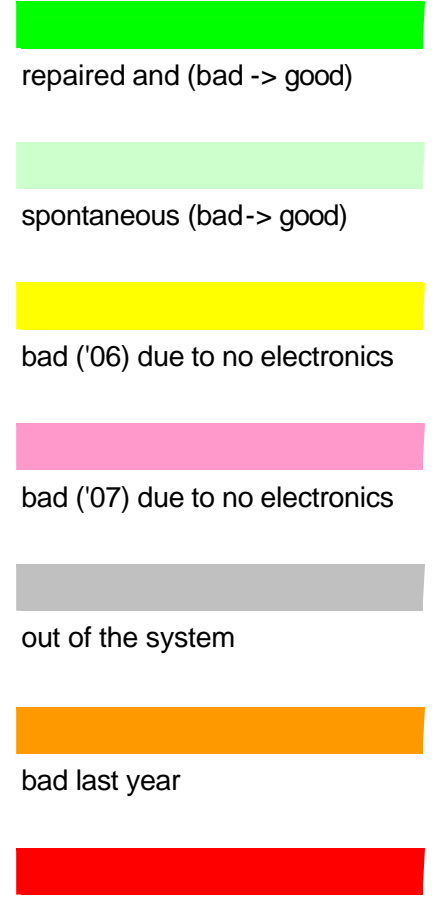

bad('07) not understood why 


\begin{tabular}{|c|c|c|c|c|c|c|c|c|c|c|c|c|}
\hline & $\mathrm{G}$ & G & $\mathrm{G}$ & $\mathrm{G}$ & $\mathrm{G}$ & $\mathrm{G}$ & $\mathrm{G}$ & G & $G$ & G & $\mathrm{G}$ & $\mathrm{G}$ \\
\hline BPM & 2_H & 2_V & 4_H & 4_V & 8_H & 8_V & 12_H & 12_V & 14_H & 14_V & 18_H & $18 \_V$ \\
\hline \multicolumn{13}{|l|}{ Bad 2006} \\
\hline \multicolumn{13}{|l|}{ No Chassis '06 } \\
\hline \multicolumn{13}{|l|}{ changes made } \\
\hline \multicolumn{13}{|l|}{ Bad 2007} \\
\hline \multirow{2}{*}{ No Chassis '07 } & & & & & & & & & $x$ & $x$ & & \\
\hline & $\mathrm{H}$ & $\mathrm{H}$ & $\mathrm{H}$ & $\mathrm{H}$ & $\mathrm{H}$ & $\mathrm{H}$ & $\mathrm{H}$ & $\mathrm{H}$ & $\mathrm{H}$ & $\mathrm{H}$ & $\mathrm{H}$ & $\mathrm{H}$ \\
\hline BPM & 2_H & 2_V & 4_H & 4_V & 8_H & 8_V & 12_H & 12_V & 14_H & 14_V & 18_H & $18 \_V$ \\
\hline \multicolumn{13}{|l|}{ Bad 2006} \\
\hline \multicolumn{13}{|l|}{ No Chassis '06 } \\
\hline \multicolumn{13}{|l|}{ changes made } \\
\hline \multicolumn{13}{|l|}{ Bad 2007} \\
\hline \multirow[t]{2}{*}{ No Chassis '07 } & & & & & & & & & $x$ & $\mathrm{x}$ & & \\
\hline & 1 & 1 & 1 & 1 & 1 & 1 & 1 & 1 & 1 & 1 & 1 & I \\
\hline BPM & 2_H & 2_V & 4_H & 4_V & 8_H & 8_V & 12_H & 12_V & 14_H & 14_V & 18_H & $18 \_\mathrm{V}$ \\
\hline \multicolumn{13}{|l|}{ Bad 2006} \\
\hline \\
\hline \multicolumn{13}{|l|}{ changes made } \\
\hline \multicolumn{13}{|l|}{$\begin{array}{l}\text { Bad } 2007 \\
\text { No Chassis '07 }\end{array}$} \\
\hline No Chassis '07 & $\mathrm{J}$ & $\mathrm{J}$ & $\mathrm{J}$ & $\mathrm{J}$ & $\mathrm{J}$ & $\mathrm{J}$ & $\mathrm{J}$ & $\mathrm{J}$ & $\mathrm{J}$ & $\mathrm{J}$ & $\mathrm{J}$ & $\mathrm{J}$ \\
\hline BPM & 2_H & 2_V & 4_H & 4_V & 8_H & 8_V & 12_H & 12_V & 14_H & 14_V & 18_H & $18 \_V$ \\
\hline Bad 2006 & $x$ & $x$ & & & & & & & & $x$ & & \\
\hline \multicolumn{13}{|l|}{ No Chassis '06 } \\
\hline \multicolumn{13}{|l|}{ changes made } \\
\hline Bad 2007 & \multicolumn{12}{|c|}{ Bad 2007} \\
\hline & & $\mathrm{K}$ & $\mathrm{K}$ & $\mathrm{K}$ & K & $\mathrm{K}$ & $\mathrm{K}$ & $\mathrm{K}$ & $\mathrm{K}$ & $\mathrm{K}$ & $\mathrm{K}$ \\
\hline BPM & 2_H & 2_V & 4_H & 4_V & 8_H & 8_V & 12_H & 12_V & 14_H & $14 \_\mathrm{V}$ & 18_H & $18 \_\mathrm{V}$ \\
\hline Bad 2006 & & & & & & & & & & & $x$ & \\
\hline \multicolumn{13}{|l|}{ No Chassis '06 } \\
\hline \multicolumn{13}{|l|}{ changes made } \\
\hline \multirow{3}{*}{$\begin{array}{l}\text { Bad } 2007 \\
\text { No Chassis '07 }\end{array}$} & & & & & & & & & & & & \\
\hline & & & $x$ & $x$ & & & & & & & & \\
\hline & L & L & L & L & $\mathrm{L}$ & $\mathrm{L}$ & $\mathrm{L}$ & L & $\mathrm{L}$ & L & L & $\mathrm{L}$ \\
\hline BPM & 2_H & 2_V & 4_H & 4_V & 8_H & 8_V & 12_H & 12_V & 14_H & 14_V & 18_H & $18 \_V$ \\
\hline \multicolumn{13}{|l|}{ Bad 2006} \\
\hline No Chassis '06 & & & & & & & & & & & & \\
\hline changes made & & & & & & & & & & & & \\
\hline Bad 2007 & & & & & & & & & & & & \\
\hline No Chassis '07 & & & & & & & & & & & & \\
\hline Bad 2007 & & & & & & & & & & & & \\
\hline
\end{tabular}




\section{Appendix VIII \\ References}

References for the scalar boards and other electronics in Bldg 929:

http://dwg-server.c-ad.bnl.gov/eng-arch/ags/electrical/d09/d09_e2634_bm.xls See drawings D09-E-2634 to D09-E-2638

http://www.cadops.bnl.gov/Hardware/BNLonly/drawings/ags/BPM_929_racks.pdf For an overview of the racks used

http://www.cadops.bnl.gov/Hardware/BNLonly/drawings/ags/BPM_929_all.pdf To see how the racks are connected

Other references mentioned in the text:

"Beam Position Monitoring", Robert Shafer, AIP Conference Proceedings 212: Accelerator Instrumentation, NY 1989

“AGS \& Booster System Random Bits of Information”, Kevin Smith, 10/9/03 See Appendix V

“AGS PUE Geometry Factor and Normalization”, Leif Ahrens, AGS Tech Note No. 459, 1997 